\title{
Characterization of mitochondrial DNA quantity and quality in the human aged and Alzheimer's disease brain
}

Hans-Ulrich Klein ${ }^{1,2^{*}}$ (D), Caroline Trumpff ${ }^{3}$, Hyun-Sik Yang ${ }^{4}$, Annie J. Lee ${ }^{2}$, Martin Picard ${ }^{3,5}$, David A. Bennett ${ }^{6}$ and Philip L. De Jager ${ }^{1,2^{*}}$

\begin{abstract}
Background: Mitochondrial dysfunction is a feature of neurodegenerative diseases, including Alzheimer's disease (AD). Changes in the mitochondrial DNA copy number (mtDNAcn) and increased mitochondrial DNA mutation burden have both been associated with neurodegenerative diseases and cognitive decline. This study aims to systematically identify which common brain pathologies in the aged human brain are associated with mitochondrial recalibrations and to disentangle the relationship between these pathologies, mtDNAcn, mtDNA heteroplasmy, aging, neuronal loss, and cognitive function.

Methods: Whole-genome sequencing data from $n=1361$ human brain samples from 5 different regions were used to quantify mtDNAcn as well as heteroplasmic mtDNA point mutations and small indels. Brain samples were assessed for 10 common pathologies. Annual cognitive test results were used to assess cognitive function proximal to death. For a subset of samples, neuronal proportions were estimated from RNA-seq profiles, and mass spectrometry was used to quantify the mitochondrial protein content of the tissue.

Results: mtDNAcn was $7-14 \%$ lower in AD relative to control participants. When accounting for all 10 common neuropathologies, only tau was significantly associated with lower mtDNAcn in the dorsolateral prefrontal cortex. In the posterior cingulate cortex, TDP-43 pathology demonstrated a distinct association with mtDNAcn. No changes were observed in the cerebellum, which is affected late by pathologies. Neither age nor gender was associated with mtDNAcn in the studied brain regions when adjusting for pathologies. Mitochondrial content and mtDNAcn independently explained variance in cognitive function unaccounted by pathologies, implicating complex mitochondrial recalibrations in cognitive decline. In contrast, mtDNA heteroplasmy levels increased by $1.5 \%$ per year of life in the cortical regions, but displayed no association with any of the pathologies or cognitive function.
\end{abstract}

\footnotetext{
* Correspondence: hk2948@cumc.columbia.edu;

pld2115@cumc.columbia.edu

'Center for Translational \& Computational Neuroimmunology, Department of

Neurology, Columbia University Irving Medical Center, New York, NY 10032,

USA

Full list of author information is available at the end of the article
}

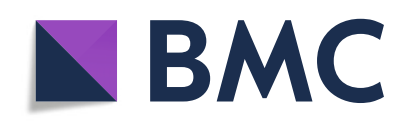

(- The Author(s). 2021 Open Access This article is licensed under a Creative Commons Attribution 4.0 International License, which permits use, sharing, adaptation, distribution and reproduction in any medium or format, as long as you give appropriate credit to the original author(s) and the source, provide a link to the Creative Commons licence, and indicate if changes were made. The images or other third party material in this article are included in the article's Creative Commons licence, unless indicated otherwise in a credit line to the material. If material is not included in the article's Creative Commons licence and your intended use is not permitted by statutory regulation or exceeds the permitted use, you will need to obtain permission directly from the copyright holder. To view a copy of this licence, visit http://creativecommons.org/licenses/by/4.0/. The Creative Commons Public Domain Dedication waiver (http://creativecommons.org/publicdomain/zero/1.0/) applies to the data made available in this article, unless otherwise stated in a credit line to the data. 
Conclusions: We studied mtDNA quantity and quality in relation to mixed pathologies of aging and showed that tau and not amyloid- $\beta$ is primarily associated with reduced mtDNAcn. In the posterior cingulate cortex, the association of TDP-43 with low mtDNAcn points to a vulnerability of this region in limbic-predominant age-related TDP-43 encephalopathy. While we found low mtDNAcn in brain regions affected by pathologies, the absence of associations with mtDNA heteroplasmy burden indicates that mtDNA point mutations and small indels are unlikely to be involved in the pathogenesis of late-onset neurodegenerative diseases.

Keywords: Mitochondria, Neurodegeneration, Alzheimer's disease, Mitochondrial DNA copy number, Mitochondrial heteroplasmy, Tau, Amyloid, TDP-43

\section{Background}

Mitochondria are complex multi-functional organelles involved in various pathways including fatty acid and cholesterol synthesis, apoptosis, calcium signaling, and adenosine triphosphate generation [1, 2]. Dysfunctional mitochondria have been described in aging [3] and in many neurodegenerative diseases such as Alzheimer's disease (AD) and amyotrophic lateral sclerosis (ALS) [4]. Mitochondria harbor their own circular genome of 16,569 base pairs encoding 13 proteins of the respiratory chain. Mitochondrial DNA (mtDNA) can be replicated independent of the cell cycle. Since mtDNA expression is required for respiratory activity, the mtDNA copy number (mtDNAcn) within a cell is regulated to meet the cell's metabolic needs, resulting in a wide range of mtDNAcn in different tissues and conditions $[5,6]$. Like nuclear DNA, mtDNA can also carry mutations, which either affect all copies of the mtDNA in a cell (termed homoplasmy) or only a fraction of the mtDNA molecules (termed heteroplasmy). Heteroplasmic mutations are assumed to be somatically generated or inherited as low level variants, and they can clonally expand over an individual's life-time [7].

The mtDNAcn has become a popular potential marker of mitochondrial health in translational studies, because mtDNAcn can be measured in stored biospecimens at large scale using qPCR or DNA sequencing techniques. In $\mathrm{AD}$, several studies have investigated mtDNAcn in tissue homogenates from different brain regions and found either a lower mtDNAcn in $\mathrm{AD}$ or no significant changes. One of the strongest reductions (50\%) was reported by an early study of the frontal cortex [8]. Smaller effect sizes or non-significant changes were reported for the hippocampus, cerebellar cortex, and cerebellum, indicating the possibility of brain region-specific effects [9-11]. Similar results were reported for other neurodegenerative diseases [5]. Interestingly, although mtDNAcn derived from whole blood is often confounded by variation in cell type composition between individuals, a recent study found an association with AD suggesting that the mtDNAcn in whole blood could potentially reflect metabolic health across tissues [12]. While the mtDNAcn overall seems to be reduced in brain regions affected by neurodegenerative diseases, mixed results have been reported for the effect of aging on mtDNAcn. For example, two studies found no evidence for agerelated changes of mtDNAcn in three brain regions, skeletal muscle, and heart muscle [13, 14], whereas a more recent study reported a decrease in skeletal muscle and an increase in liver tissue with age [15].

A higher burden of mtDNA heteroplasmy has been observed in tissues from aged individuals [16]. Increased levels of heteroplasmic mtDNA deletions as well as heteroplasmic point mutations were also described in brains from $\mathrm{AD}$ and Parkinson's disease (PD) patients $[8,17,18]$, which lead to the hypothesis that pathogenic mtDNA mutations, when they exceed certain thresholds, could contribute to the mitochondrial dysfunction observed in late-onset neurodegenerative diseases. However, a recent high-throughput sequencing study looking at heteroplasmic point mutations found no evidence for an association with $\mathrm{AD}$ or PD [9].

In this study, we profiled mtDNAcn and mtDNA heteroplasmy levels in $n=762$ brain samples from the Religious Orders Study and the Rush Memory and Aging Project (ROSMAP) [19] and implemented substantial improvements compared to previous studies: (i) The detailed pathologic characterization of ROSMAP samples facilitated the disentanglement of the effects of different brain pathologies and aging on mtDNA. Mixed pathologies are common in aged individuals including $\mathrm{AD}$ patients [20] and unaccounted pathologies may have contributed to some of the mixed results in the literature. (ii) Standardized cognitive tests conducted proximate to death were employed to assess the association between cognitive functioning and mtDNAcn adjusted for pathologies. (iii) Using RNA-seq-derived estimations of cell type proportions, we accounted for neuronal loss as a major confounder of mtDNAcn analyses in $\mathrm{AD}$ brains. (iv) We profiled five different brain regions to assess brain-regional differences (three regions in ROSMAP, and two additional regions in two independent datasets with a total of $n=599$ additional samples). (v) Finally, we calculated a proteomic score representing mitochondrial content to investigate whether changes in 
mtDNAcn reflect changes of mitochondrial mass or whether they are specific to mtDNA maintenance.

\section{Methods \\ ROSMAP cohort and neuropathologic characterization}

The Religious Orders Study (ROS) and the Rush Memory and Aging Project (MAP) are two cohort studies of aging and dementia conducted by the same team of investigators and share a large common core of harmonized clinical and post-mortem data collection which allows for joint analyses [19]. Participants entered the studies without known dementia and agreed to annual clinical and cognitive assessments as well as brain donation after death.

To obtain a pathologic diagnosis of $\mathrm{AD}$, a modified Bielschowsky silver stain was used to visualize neuritic plaques, diffuse plaques, and neurofibrillary tangles in five cortical regions (hippocampus, entorhinal, midfrontal, middle temporal, and inferior parietal). A board-certified neuropathologist, blinded to clinical data, determined the pathologic diagnosis of $\mathrm{AD}$ based on an intermediate to high likelihood of $\mathrm{AD}$ according to the NIA Reagan criteria. A quantitative global AD pathology score was derived from the Bielschowsky silver stains by counting and standardizing each of the pathologies neuritic plaques, diffuse plaques, and neurofibrillary tangles in each of the five cortical regions, and then averaging the standardized measures across regions. The average of these three pathology measures was then used as single score of AD pathology burden. Amyloid- $\beta$ and tau tangles were assessed in 8 brain regions (hippocampus, entorhinal cortex, midfrontal cortex, inferior temporal gyrus, angular gyrus, calcarine cortex, anterior cingulate cortex, and superior frontal cortex) using immunochemistry [21, 22]. Paraffin-embedded sections were immunostained for amyloid- $\beta$ using 1 of 3 monoclonal anti-human antibodies: 4G8 (1:9000; Covance Labs, Madison, WI), 6F/3D (1:50; Dako North America Inc., Carpinteria, CA), and 10D5 (1:600; Elan Pharmaceuticals, San Francisco, CA). Paired helical filament (PHF) tau tangles were labeled with an antibody specific to phosphorylated tau (AT8, Thermoscientific, Waltham, MA, USA). A computerized sampling procedure combined with image analysis software was used to calculate the percentage area occupied with amyloid- $\beta$ and the density of PHFtau tangles. Composite scores were computed for overall amyloid- $\beta$ burden and tau tangle density by averaging the scores obtained from the eight brain regions. The three quantitative measurements (global AD pathology, amyloid- $\beta$, and tau) were square root transformed for better statistical properties.

Presence of TDP-43 cytoplasmic inclusions in neurons and glia were determined in eight regions (amygdala, entorhinal cortex, hippocampus CA1, hippocampus dentate gyrus, anterior temporal pole cortex, midtemporal cortex, orbital frontal cortex, and midfrontal cortex) using a phosphorylated monoclonal TAR5P-1D3 (pS409/410; 1:100, Ascenion, Munich, Germany) TDP43 antibody. Based on the absence or presence of TDP43 pathology in the eight regions, four stages of TDP-43 distribution were recognized: none, amygdala, amygdala + limbic, amygdala + limbic + neocortical [23]. Lewy body disease was assessed in four stages (not present, nigral-predominant, limbic-type, neocortical-type). Seven regions (substantia nigra, anterior cingulate cortex, entorhinal cortex, amygdala, midfrontal cortex, superior or middle temporal cortex, inferior parietal cortex) were assessed for Lewy bodies using $\alpha$-synuclein immunostaining (LB509; 1:150 or 1:100, Zymed Labs, Invitrogen, Carlsbad, CA, USA; and pSyn\#64; 1:20,000; Wako Chemicals, Richmond, VA,USA) [24]. Cerebral amyloid angiopathy pathology was assessed in four neocortical regions (midfrontal, midtemporal, parietal, and calcarine cortices) using using 1 of 3 monoclonal anti-human antibodies: 4G8 (1:9000; Covance Labs, Madison, WI), 6F/3D (1:50; Dako North America Inc., Carpinteria, CA), and 10D5 (1:600; Elan Pharmaceuticals, San Francisco, CA). Similar to the protocol by Love, Chalmers [25], meningeal and parenchymal vessels were assessed for amyloid- $\beta$ deposition in each region and scored from 0 to 4 . Scores were averaged across the four regions and categorized into none, mild, moderate, or severe [26]. Large vessel cerebral atherosclerosis rating was made by visual inspection after paraformaldehyde fixation, at the Circle of Willis at the base of the brain, and included evaluation of the vertebral, basilar, posterior cerebral, middle cerebral, and anterior cerebral arteries and their proximal branches. Severity was graded (none or possible, mild, moderate, severe) by visual examination of the extent of involvement of each artery and number of arteries involved [27]. Arteriolosclerosis was graded by evaluating the vessels of the anterior basal ganglia for histological changes as previously described [28]. Four stages (none, mild, moderate, and severe) were recognized. The presence of one or more gross chronic cerebral infarctions was determined during gross examination and confirmed histologically. The presence of one or more chronic microinfarcts was determined on sections of a minimum of nine regions stained with hematoxylin and eosin (H\&E) [29]. The presence of hippocampal sclerosis was identified by severe neuronal loss and gliosis on H\&E-stained sections in CA1 or subiculum [30].

DNA for whole-genome sequencing (WGS) was extracted from the dorsolateral prefrontal cortex (DLPFC) using Qiagen's QIAamp DNA kit $(n=367)$ or Qiagen's AllPrep Universal kit $(n=87)$, from the posterior cingulate cortex (PCC) using Qiagen's AllPrep Universal kit 
( $n=66$ ), or from the cerebellum (CB) using Qiagen's Gentra Puregene Tissue kit $(n=242)$. Sample characteristics are summarized in Table 1.

\section{Mayo and MSBB cohorts}

Samples included in the Mayo case-control study were obtained either from the Mayo Brain Bank or from the Banner Sun Health Institute and classified as control, $\mathrm{AD}$, progressive supranuclear palsy (PSP) or pathologic aging based on neuropathological assessment [31]. All AD samples demonstrated tau pathology (Braak score $\geq 4$ ). All controls demonstrated no or minimal tau pathology (Braak score $\leq 3$ ) and were without any other neurodegenerative disease. In this study, samples from the Banner Sun Health Institute were excluded since all AD samples were obtained from the Mayo Brain Bank and we observed a difference in mtDNAcn between control samples from the two different centers. Since age at death was right-censored at 95 years for HIPPA compliance, we stratified age into 5 year strata with an open interval $>$ 95 years for our analyses. Specimens for WGS were sampled from the temporal cortex (TCX) $(n=262)$. Sample characteristics are summarized in Table S1.

The Mount Sinai Brain Bank (MSBB) case-control study cohort was assembled after applying stringent inclusion/exclusion criteria and represents the full spectrum of cognitive and neuropathological AD severity in the absence of discernable non-AD neuropathology [32]. Neuropathological assessments were performed according to the Consortium to Establish a Registry for Alzheimer's Disease (CERAD) protocol and included assessment by hematoxylin and eosin, modified Bielschowski, modified thioflavin $\mathrm{S}$, and anti-amyloid- $\beta$ (4G8), anti-tau (AD2) and anti-ubiquitin (Dakoa Corp.). Pathologic AD was defined based on the CERAD stages definitive AD and probable AD. Samples staged as possible AD or not AD were considered as controls. Age at death was right-censored and stratified as for the Mayo cohort. Specimens for WGS were sampled from the frontal pole (FP) $(n=337)$. Sample characteristics are summarized in Table S2. A total of $n=67$ samples were obtained from non-Caucasians and excluded from the mtDNAcn GWAS and mtDNA heteroplasmy analyses.

\section{Whole-genome sequencing}

WGS libraries from all three studies were prepared using the KAPA Hyper Library Preparation Kit in accordance with the manufacturer's instructions. Briefly, $650 \mathrm{ng}$ of DNA was sheared using a Covaris LE220 sonicator (adaptive focused acoustics). DNA fragments underwent bead-based size selection and were subsequently endrepaired, adenylated, and ligated to Illumina sequencing adapters. Final libraries were evaluated using fluorescent-based assays including $\mathrm{QPCR}$ with the Universal KAPA Library Quantification Kit and Fragment

Table 1 Characteristics of the ROSMAP cohort

\begin{tabular}{|c|c|c|c|}
\hline & DLPFC $(n=454)$ & $P C C(n=66)$ & $C B(n=242)$ \\
\hline Sex (male) & $158(34.8 \%)$ & $21(31.8 \%)$ & $71(29.3 \%)$ \\
\hline Age (years) & $89.3(6.6)$ & $89.1(5.6)$ & $88(6.7)$ \\
\hline Pathologic AD & $308(67.8 \%)$ & $45(68.2 \%)$ & $141(58.3 \%)$ \\
\hline Amyloid (\% area affected) & $4.6(4.4)$ & $3.9(3.8)$ & $4(4.3)$ \\
\hline Tau (\% area affected) & $7.4(8.1)$ & $6.8(6.8)$ & $6.2(7.9)$ \\
\hline TDP-43 (none, amygdala, limbic, neocortical) & $\begin{array}{l}205,76,97,44(48.6,18.0,23.0 \\
10.4 \%)\end{array}$ & $\begin{array}{l}23,16,12,12(36.5,25.4,19.0 \\
19.0 \%)\end{array}$ & $\begin{array}{l}96,45,38,33(45.3,21.2,17.9 \\
15.6 \%)\end{array}$ \\
\hline Lewy bodies (none, nigral, limbic, neocortical) & $\begin{array}{l}337,7,32,60(77.3,1.6,7.3 \\
13.8 \%)\end{array}$ & $48,2,2,9(78.7,3.3,3.3,14.8 \%)$ & $\begin{array}{l}184,6,21,25(78.0,2.5,8.9 \\
10.6 \%)\end{array}$ \\
\hline $\begin{array}{l}\text { Cerebral amyloid angiopathy (none, mild, } \\
\text { moderate, severe) }\end{array}$ & $\begin{array}{l}92,191,110,52(20.7,42.9,24.7 \\
11.7 \%)\end{array}$ & $\begin{array}{l}13,25,16,11(20.0,38.5,24.6 \\
16.9 \%)\end{array}$ & $\begin{array}{l}58,101,51,24(24.8,43.2,21.8, \\
10.3 \%)\end{array}$ \\
\hline $\begin{array}{l}\text { Cerebral atherosclerosis (none, mild, moderate, } \\
\text { severe) }\end{array}$ & $\begin{array}{l}84,212,123,33(18.6,46.9,27.2 \\
7.3 \%)\end{array}$ & $\begin{array}{l}11,25,19,11(16.7,37.9,28.8 \\
16.7 \%)\end{array}$ & $\begin{array}{l}39,114,72,15(16.2,47.5,30.0, \\
6.2 \%)\end{array}$ \\
\hline Arteriolosclerosis (none, mild, moderate, severe) & $\begin{array}{l}136,157,118,42(30.0,34.7 \\
26.0,9.3 \%)\end{array}$ & $\begin{array}{l}9,26,22,9(13.6,39.4,33.3 \\
13.6 \%)\end{array}$ & $\begin{array}{l}78,79,65,17(32.6,33.1,27.2 \\
7.1 \%)\end{array}$ \\
\hline Gross chronic infarcts (one or more) & $171(37.7 \%)$ & $22(33.3 \%)$ & $83(34.3 \%)$ \\
\hline Chronic microinfarcts (one or more) & $132(29.1 \%)$ & $18(27.3 \%)$ & $70(28.9 \%)$ \\
\hline Hippocampal sclerosis (present) & $39(8.7 \%)$ & $6(9.2 \%)$ & $22(9.2 \%)$ \\
\hline Post mortem interval (hours) & $8.4(5.7)$ & $6.6(4.7)$ & $8.3(6.7)$ \\
\hline
\end{tabular}

Column headers denote the brain regions where specimens for WGS were sampled. Reported pathology burdens were obtained by considering multiple regions (see methods) and are not specific to the brain region selected for WGS. Mean and standard deviation are shown for continuous variables. Absolute frequency and percentage are shown for categorical variables 
Analyzer (Advanced Analytics) or BioAnalyzer (Agilent 2100). Libraries were sequenced on an Illumina HiSeq $X$ sequencer (v2.5 chemistry) using $2 \times 150 \mathrm{bp}$ cycles.

\section{Variant calling}

Sequence alignment and nuclear DNA variant calling was performed by the automated pipeline of the New York Genome Center, where all samples were sequenced [33]. Briefly, paired-end 150 bp reads were aligned to the GRCh37 human reference using the Burrows-Wheeler Aligner (BWA-MEM v0.7.8) and processed using the GATK best-practices workflow that includes marking of duplicate reads using Picard tools v1.83, local realignment around indels, and base quality score recalibration (BQSR) using the Genome Analysis Toolkit (GATK v3.4.0).

Mitochondrial homo- and heteroplasmic singlenucleotide variants and small indels $(<50 \mathrm{bp})$ were called following GATK's Best Practices Mitochondria Pipeline 1.1.0 (https://github.com/gatk-workflows/gatk4mitochondria-pipeline). GATK v4.1.2 was used to run the pipeline with the rCRS (NC_012920.1) as mtDNA reference sequence. Briefly, sequence reads (mapped to MT or unmapped in original bam file) were mapped to the rCRS and to the rCRS shifted by 8000 base pairs using BWA-MEM v0.7.8. Variants were detected using GATK's Mutect2 in both bam files. Subsequently, variants were merged into one VCF file using the shifted rCRS for variants around the artificial start/end position of the circular genome and the unmodified rCRS for the remaining part the MT genome. Variant filtering included the median autosomal chromosome coverage to filter potential polymorphic nuclear mitochondrial DNA (NUMT) variants, the mtDNA contamination estimated by the haplochecker (mitolib 0.1.2) to account for possible contamination, a minimum minor allele frequency of 0.03 , and an F-score beta of 1 (default settings).

\section{Estimation of the mtDNAcn from WGS data}

The median sequence coverages of the autosomal chromosomes $\operatorname{cov}_{n u c}$ and of the mitochondrial genome $\operatorname{cov}_{m t}$ were calculated using R/Bioconductor (packages GenomicAlignments and GenomicRanges). Ambiguous regions were excluded using the intra-contig ambiguity mask from the BSgenome package. The mtDNAcn was defined as $\left(\operatorname{cov}_{m t} / \operatorname{cov}_{\text {nuc }}\right) \times 2$. Raw mtDNAcn was used for the first analysis shown in Fig. 1. For subsequent analyses the mtDNAcn was $z$-standardized within each brain region and DNA extraction kit and then logarithmized. The normalization facilitated the combined analysis of the two different kits used for the DLPFC and resulted in approximately normal mtDNAcn measures (Fig. S1).
Estimation of the neuronal proportion from RNA-seq data Proportion of neurons were estimated for $n=327$ DLPFC samples with RNA-seq data by applying the Digital Sorting Algorithm (DSA) [34] to a set of published marker genes that were previously used to deconvolute cortical RNA-seq data [35]. RNA-seq data were TMM normalized and technical variables were regressed out. Only marker genes with a mean transcription level $\geq 2 \mathrm{cpm}$ in our dataset were used ( 87 markers for astrocytes, 88 for endothelial cells, 59 for microglia, 90 for neurons, and 86 for oligodendrocytes). As proposed by Wang, Allen [35], DSA was modified so that the median instead of the mean transcription level of all marker genes per cell type was calculated.

\section{Estimation of mitochondrial content from proteomic data} Tandem mass tag (TMT) multiplexed mass spectrometry data was available for a subset of $n=156$ DLPFC samples that also had WGS data. Mass spectrometry data was preprocessed and normalized as previously described [36]. Ten proteins were selected to quantify mitochondrial mass. Six proteins (CS, LRPPRC, SLC25A24, TIMM44, GCDH, and TRAP1) were taken from the Human Protein Atlas' list of mitochondrial marker proteins [37]. The four additional proteins (HSPD1, VDAC2, VDAC3, and TOMM20) have been previously used as mitochondrial markers and confirmed to be specific to mitochondria in the Human Protein Atlas. Fig. S5A shows the correlation between the 10 selected proteins. The median protein level of these ten proteins was used as mitochondrial content measure. Similarly, the respiratory chain complexes I-V were quantified by calculating the median level of all proteins that were detected in our TMT data and were known subunits or assembly factors of the respective complex. Supplementary Excel File 3 lists the proteins used to calculate the scores. This list includes 11 of the 13 mtDNA-encoded proteins (see column C in Supplementary Excel File 3) and their median protein level was used to specifically quantify the abundance of mtDNAencoded proteins.

\section{Statistical methods}

Statistical analyses were conducted in R. Standard multivariable linear regression models were used when the standardized mtDNAcn or cognitive function was the dependent variable. Quasi-Poisson regression models were used when the number of mtDNA heteroplasmic mutations (heteroplasmy level) was the dependent variable. Reported $p$ values were obtained from likelihood ratio tests and unadjusted unless stated otherwise in the text. Continuous variables were standardized to obtain comparable effect sizes unless stated otherwise. All variables included in the respective models are described in 

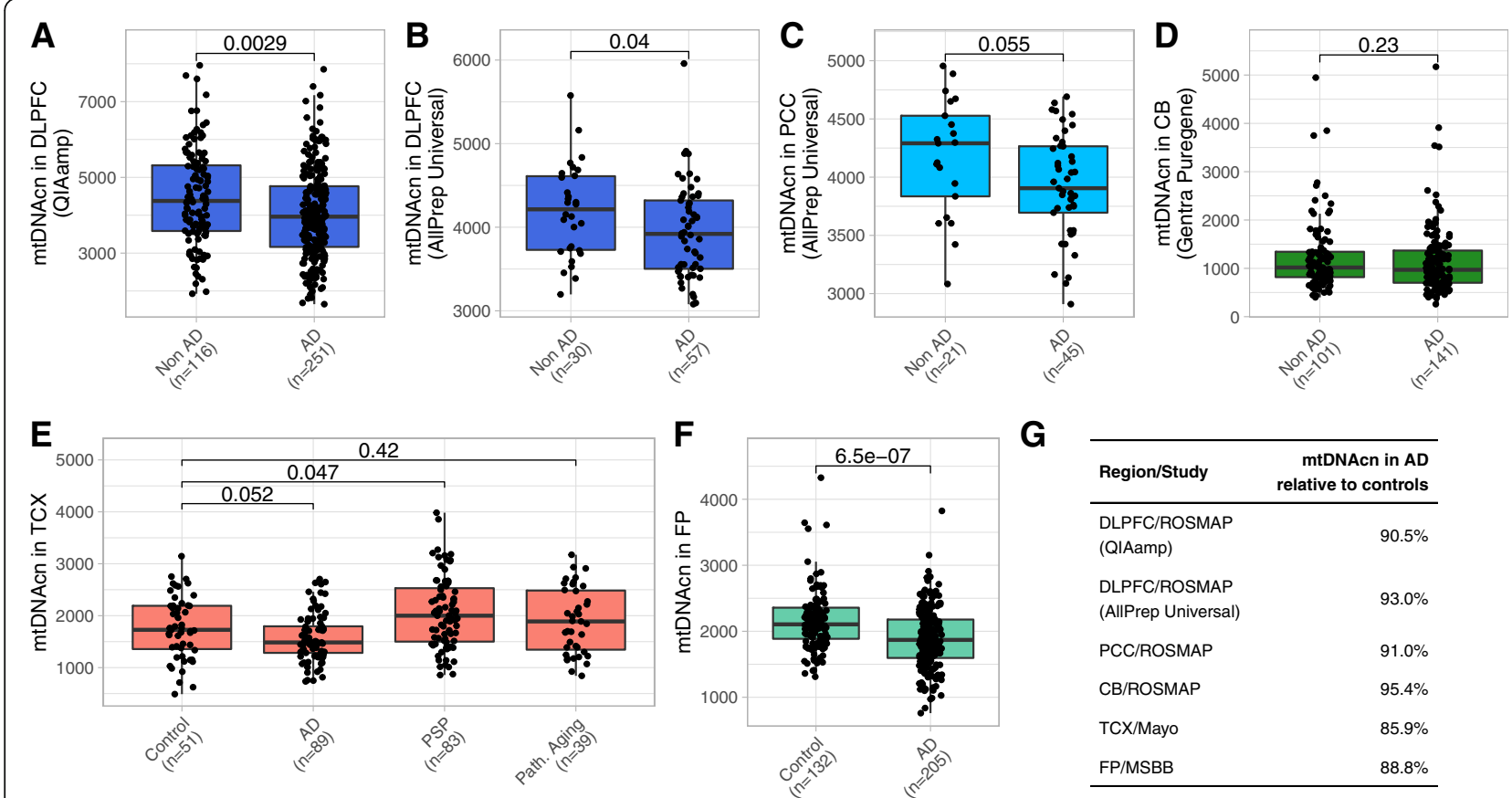

\begin{tabular}{lr}
\hline Region/Study & $\begin{array}{r}\text { mtDNAcn in AD } \\
\text { relative to controls }\end{array}$ \\
\hline $\begin{array}{l}\text { DLPFC/ROSMAP } \\
\text { (QIAamp) }\end{array}$ & $90.5 \%$ \\
$\begin{array}{l}\text { DLPFC/ROSMAP } \\
\text { (AllPrep Universal) }\end{array}$ & $93.0 \%$ \\
PCC/ROSMAP & $91.0 \%$ \\
CB/ROSMAP & $95.4 \%$ \\
TCX/Mayo & $85.9 \%$ \\
FP/MSBB & $88.8 \%$ \\
\hline
\end{tabular}

Fig. 1 The mtDNAcn is reduced in cortical brain regions in $A D$. (A-D) Boxplots comparing the mtDNAcn in the DLPFC (A and B), in the PCC (C), and in the $C B(D)$ from individuals of the ROSMAP cohort with and without pathologic AD diagnosis. The used DNA extraction kit is denoted in brackets on the $y$ axis. Wilcoxon rank-sum test was applied to calculate $p$ values. (E) Boxplot shows the mtDNAcn in the TCX of controls, AD cases, PSP cases, and cases of pathologic aging from the Mayo study. Wilcoxon rank-sum test was applied to calculate $p$ values. (F) Boxplot shows the mtDNAcn in the FP of controls and AD cases from the MSBB study. Wilcoxon rank-sum test was applied to calculate $p$ values. (G) The estimated relative mtDNAcn observed in AD compared to controls is shown for each brain region and study

the text and figures. When analyzing the effect of SNPs on mtDNAcn, population structure was modeled by the first three principle components obtained from the genotypes after pruning. Random effects meta-analyses were performed using the $\mathrm{R}$ package meta v4.13. $P$ values and quasi-Bayesian confidence intervals for the mediation analysis were calculated using the $\mathrm{R}$ package mediation v4.5.0 [38]. The sparse Gaussian graphical model (Fig. 5B) was estimated by the graphical LASSO method with EBIC model selection and a default tuning parameter of 0.5 as implemented in the R package bootnet [39]. The nonparanormal transformation implemented in the package huge was applied to relax the assumption of normality for the mtDNA heteroplasmy levels before estimating the network. Network stability was assessed using 1000 bootstraps.

\section{Results}

\section{mtDNAcn is reduced in the AD cortex}

Whole genome sequencing (WGS) data were generated from $n=762$ post-mortem brains from ROSMAP, two harmonized cohort studies of aging and dementia [19]. Brain specimens were obtained from the dorsolateral prefrontal cortex (DLPFC) $(n=454)$, the posterior cingulate cortex (PCC) $(n=66)$, and the cerebellum (CB) $(n=242)$ from different individuals. Table 1 summarizes pathologic and demographic information of the samples. The number of aligned sequence reads per sample ranged from $643.5 \times 10^{6}$ to $1475.0 \times 10^{6}$ reads (median of $899.1 \times 10^{6}$ reads) (Supplementary Excel File 1). The mtDNAcn was estimated from the WGS data by dividing the median coverage of the MT chromosome by the median coverage of the autosomal chromosomes and multiplying the ratio by two [40, 41].

We first studied whether the mtDNAcn in the three selected brain regions was altered in individuals with pathologic $\mathrm{AD}$ diagnosis compared to those without $\mathrm{AD}$ (Fig. 1A-D). Two different DNA extraction kits were used for the DLPFC samples. Since the DNA extraction method can influence the mtDNAcn estimates [42, 43], the DLPFC samples were split by kit and analyzed separately. In line with previous reports $[8,9]$, we found a lower mtDNAcn in AD in the DLPFC $(p=0.0029$ and $p=0.040$ for the two kits/batches, Wilcoxon rank-sum tests) and also in the PCC $(p=0.055$, Wilcoxon ranksum test). Changes in the PCC were of similar magnitude but failed to reach significance probably due to the smaller sample size. Changes in the $C B$ were not significant. 
Next, we analyzed the mtDNAcn in two additional brain regions using public WGS data from the Mayo Clinic study and the Mount Sinai Brain Bank (MSBB) study (Tables S1,S2). Both regions, the temporal cortex (TCX) in the Mayo data ( $p=0.052$, Wilcoxon test) and the frontal pole (FP) in the MSBB data $\left(p=6.5 \times 10^{-7}\right.$, Wilcoxon test), demonstrated a lower mtDNAcn in AD samples compared to control samples (Fig. 1E,F). The reduction of mtDNAcn in $\mathrm{AD}$ ranged from 7.0 to $14.2 \%$ across the cortical regions from the three studies (Fig. 1G). The Mayo study includes samples diagnosed with pathologic aging, which is characterized by amyloid- $\beta$ loads at similar levels as in $\mathrm{AD}$ and an absence of tau pathology. Whether pathologic aging is an early stage of $\mathrm{AD}$ or whether these individuals have protective factors that prevent the development of tau pathology is unknown [44]. We did not observe altered mtDNAcn levels in the TCX of individuals with pathologic aging (Fig. 1E). Additionally, the Mayo study includes samples with progressive supranuclear palsy (PSP). PSP is a tauopathy primarily characterized by tau inclusions in the brain stem and subcortical neurons. Interestingly, we observed a moderately higher mtDNAcn in the TCX of the PSP samples compared to the control samples $(p=0.047$, Wilcoxon rank-sum test) (Fig. 1E).

For the subsequent analyses, we log-transformed the raw mtDNAcn and calculated $\mathrm{z}$-scores for each of the six datasets shown in Fig. 1A-F. Then, we merged the two ROSMAP DLPFC datasets generated by different DNA extraction kits and verified that the normalized mtDNAcn approximately follows a standard normal distribution (Fig. S1). We note that the raw mtDNAcn before normalization should also be considered as a relative rather than an absolute measurement since the estimations are likely affected by experimental factors, which impedes a comparison across different brain regions or studies when different protocols and reagents were used.

\section{Lower mtDNAcn is primarily related to tau in the DLPFC and to TDP-43 in the PCC}

$\mathrm{AD}$ pathology is the most common brain pathology in the aged brain but is often accompanied by comorbid brain pathologies. To determine which pathological feature is driving the association with mtDNAcn, we first analyzed each of the 12 pathologic variables shown in Fig. 2A as well as cognition and cognitive decline separately adjusted only for age and sex. The two cognitive variables were additionally adjusted for education. Further, we also analyzed the association with age and sex adjusted for AD pathology (Fig. 2A). Detailed results of these analyses are given in Table S3 and can be summarized by four main findings: First, in the DLPFC, mtDNAcn is mainly associated with tau $\left(p=2.9 \times 10^{-6}\right.$, $\mathrm{t}$ test) rather than amyloid- $\beta$ pathology $(p=0.011, \mathrm{t}$ test $)$ and is also associated with cognition proximate to death $\left(p=7.9 \times 10^{-10}, \mathrm{t}\right.$ test $)$ and cognitive decline $(p=1.8 \times$ $10^{-9}, \mathrm{t}$ test). Second, in the PCC, we also found an association with cognition $\left(p=1.7 \times 10^{-5}\right.$, t test $)$ and cognitive decline $\left(p=5.5 \times 10^{-4}, \mathrm{t}\right.$ test), but the association with tau was not significant probably due to the smaller sample size. Interestingly, we found an association with TDP-43 pathology $\left(p=2.1 \times 10^{-3}\right.$, F test $)$ in the PCC, which explained $23 \%$ of the variance in mtDNAcn. Third, in the CB, neither pathologies nor cognitive measures were associated with mtDNAcn. Fourth, neither age nor sex were associated with mtDNAcn in any of the three regions when adjusting for AD pathology. All significant pathologies demonstrated an inverse correlation with mtDNAcn, and a lower mtDNAcn was associated with lower cognitive performance and a steeper rate of cognitive decline, consistent with the notion that a high mtDNAcn is a feature of healthy mitochondrial function in the aged brain.

Pathologic TDP-43 is known to localize inside mitochondria and has recently been described to trigger the release of mtDNA molecules from the mitochondrial matrix into the cytoplasm in cellular systems [45-47]. In our study, limbic-predominant age-related TDP-43 encephalopathy neuropathological change (LATE-NC) was captured by phosphorylated TDP-43 immunohistochemistry, and staged according to the recent consensus working group report (stage 0 , no TDP-43; stage 1, localized to amygdala; stage 2, extension to hippocampus or entorhinal cortex; stage 3, extension to the neocortex) [48]. In the PCC, we observed a gradual reduction of mtDNAcn across the stages with a distinctly lower mtDNAcn in stage 3 (Fig. 2B). In contrast, in the DLPFC we observed only a minor reduction in the latest stage (Fig. S2A). Our findings are consistent with previous reports of prominent PCC hypometabolism in LATENC [49], and DLPFC being involved only in the most advanced stage of LATE-NC $[50,51]$. Thus, mtDNAcn alterations in the PCC might be an early feature of LATE-NC, even though the current LATE-NC staging scheme does not consider the PCC.

Neurons have relatively high concentrations of mitochondria, and changes in the proportion of neurons during the course of $\mathrm{AD}$ can confound mtDNAcn measurements at the tissue level. We therefore estimated the proportions of neurons and of glial cell types from DLPFC RNA-seq data for a subset of $n=327$ samples with DLPFC mtDNAcn estimates. As expected, the neuronal proportion was significantly positively associated with mtDNAcn, accounting for $6.9 \%$ of the variance in mtDNAcn $\left(p=1.7 \times 10^{-6}\right.$, t test) (Fig. $2 \mathrm{C}$, Table S4). We also found an association with oligodendrocytes ( $p=0.013$, t test), but in contrast to the neuronal 


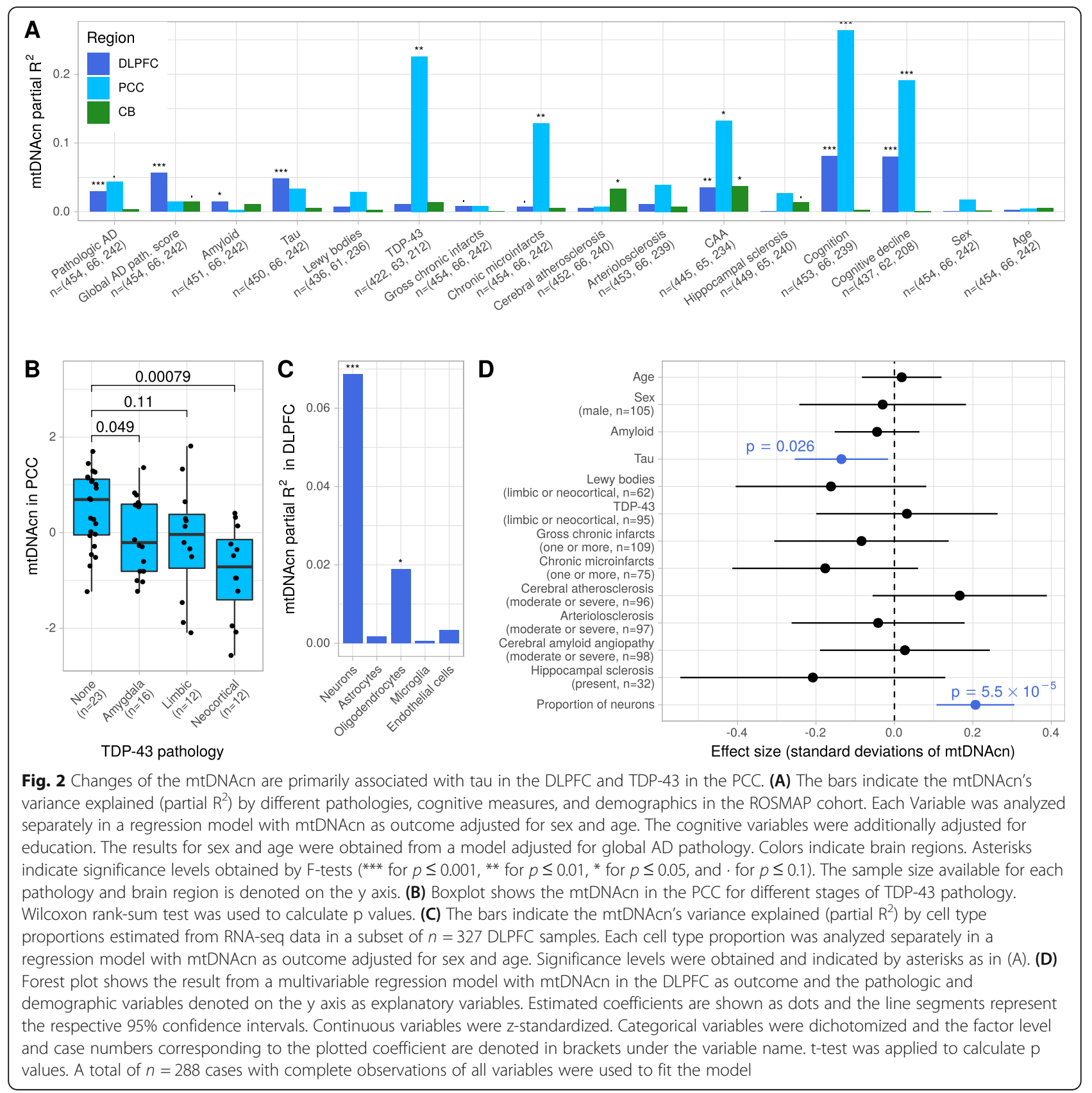

proportion this association disappeared when we modeled the different cell types together, indicating that adjusting for neuronal proportions in downstream analyses is sufficient.

Since many of the pathologies are correlated with each other, we next modeled all pathologies, age, sex and neuronal proportion in a multivariable regression model to identify the primary driver of mtDNAcn changes in the DLPFC. As shown in Fig. 2D, only tau pathology and neuronal proportion remained significantly associated with mtDNAcn when accounting for all pathologies. Thus, our data suggest that tau burden is directly associated with lower mtDNAcn in the DLPFC and that this relationship cannot be explained by neuronal loss alone. Similar results for tau were obtained from a model unadjusted for neuronal proportion (Fig. S2B).

\section{mtDNAcn is associated with cognitive function independent of brain pathologies}

The univariate analysis (Fig. 2A) revealed that a lower mtDNAcn in the cortex is associated with a decline of cognitive function. We next sought to investigate whether this relation can be explained by the correlation of mtDNAcn with tau, or whether mtDNAcn and tau 
have effects on cognitive functioning independent of each other. We modeled cognitive function as outcome depending on age, sex, education, mtDNAcn, and ten neuropathologies including tau using our DLPFC data. In this model, mtDNAcn was significantly associated with cognitive function $\left(p=2.4 \times 10^{-4}\right.$, t test) together with tau, Lewy bodies, gross chronic infarcts, hippocampal sclerosis, and education (Fig. 3A). Tau was the most important predictor for cognitive function. When we added neuronal proportion to the model, the effect size of mtDNAcn was attenuated by $26 \%$ but remained significant ( $p=0.034$, $\mathrm{t}$ test) despite the smaller number of samples that have both measurements neuronal proportion and mtDNAcn (Fig. 3B). These results indicate that the observed changes in the mtDNAcn in the DLPFC capture aspects of mitochondrial health beyond neuronal loss and correlates with cognitive function independent of pathologies.

\section{Genetic determinants of the mtDNAcn}

Two recent genome-wide association studies (GWAS) of blood samples from the UK Biobank identified 96 and 50 independent loci respectively that affected the mtDNAcn in whole blood [52, 53]. Functional annotation of some loci pointed to blood-specific pathways such as platelet activation or megakaryocyte proliferation, an association consistent with the influence of platelets on whole blood mtDNAcn [54], whereas other loci were located at genes involved in mitochondrial pathways and therefore could regulate the mtDNAcn in brain tissues as well. Since our sample size is limited for a GWAS, we conducted a focused analysis of 81 lead SNPs identified in the prior study [52] and with a minor allele frequency $\geq 5 \%$ in our data. We separately analyzed the four brain regions with at least 100 samples (DLPFC, $\mathrm{CB}$, TCX, and FP) and subsequently performed a random effects meta-analysis. A total of $n=67$ nonCaucasian individuals in the MSBB dataset (FP) were excluded resulting in $n=1228$ brain samples. The association tests were adjusted for age, sex, population structure, and quantitative AD pathology score (DLPFC, $\mathrm{CB}$ ) or post-mortem diagnosis respectively (TCX, FP). After Bonferroni adjustment, only the top SNP rs11085147 from the original study was significantly associated with mtDNAcn in our brain data $\left(\mathrm{p}_{\mathrm{BF}}=\right.$ 0.0436). The direction of the effect was identical in all four brain regions and consistent with the originally reported direction in blood. Each additional dosage of the alternative allele increased the mtDNAcn by 0.23 standard deviations. The SNP rs11085147 is a missense
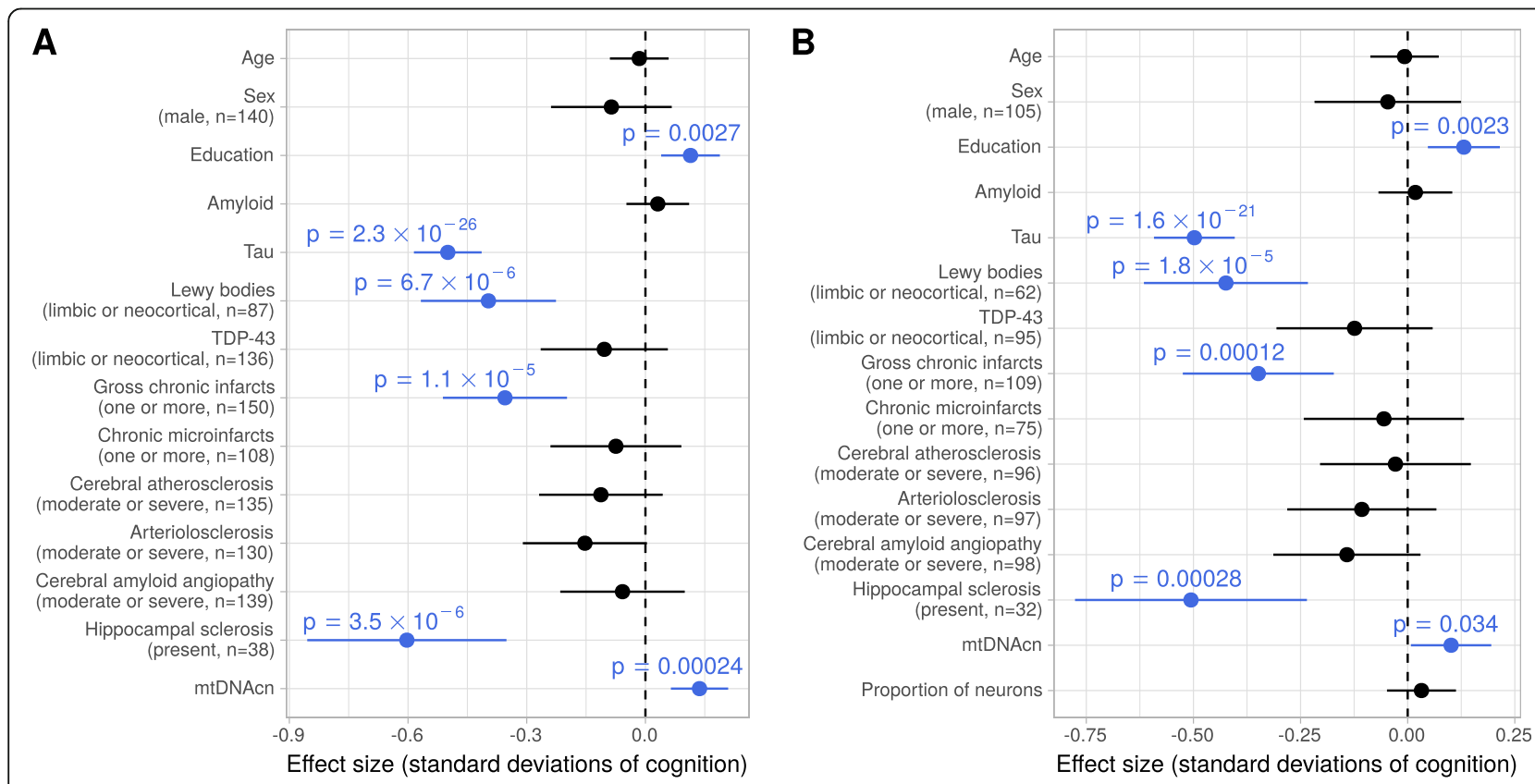

Fig. 3 The mtDNAcn is associated with cognitive function independent of brain pathologies. (A) Forest plot shows the result from a multivariable regression model with global cognitive function as outcome and the pathologic variables, demographic variables and mtDNAcn as explanatory variables (y axis). Estimated coefficients are shown as dots and the line segments represent the respective 95\% confidence intervals. Continuous variables were z-standardized. Categorical variables were dichotomized and the factor level and case numbers are denoted in brackets under the variable name. t-tests were applied to calculate $p$ values. A total of $n=393$ cases with complete observations of all variables were used to fit the model. (B) Same plot as in (A) but with neuronal proportion as additional variable in the model reducing the number of samples with complete observations to $n=287$ 
variant of the gene Lon Peptidase 1, Mitochondrial (LONP1), which binds mitochondrial DNA and is involved in mtDNA replication and mitogenesis [55]. rs11085147 has not been linked to AD risk in genomewide association studies of AD. Detailed results for all 81 SNPs are given in Supplementary Excel File 2.

The strongest genetic risk factor for late-onset $\mathrm{AD}$ is the Apolipoprotein E (APOE) locus. Several studies using mouse models or human brain implicated the $\varepsilon 4$ risk allele with mitochondrial dysfunction [56, 57]. We therefore analyzed the effect of the APOE \&4 allele on the mtDNAcn in our four datasets with $\geq 100$ samples. The APOE \&4 allele was associated with lower mtDNAcn ( $p=8.0 \times 10^{-7}$, random effects meta-analysis), but this association was attenuated considerably when adjusting for pathologies ( $p=0.014$, random effects meta-analysis) (Table 2) suggesting that a large fraction but not the complete $A P O E \& 4$ effect on mtDNAcn is mediated via AD pathology. Indeed, mediation analysis of the DLPFC samples (Fig. S3) revealed that $44 \%$ of the total effect is mediated via pathology.

mtDNA heteroplasmy levels in the cortex are higher with age

We detected the number of mtDNA heteroplasmic point mutations and small indels (less than $50 \mathrm{bp}$ ) in the WGS data to assess the relation of mtDNA mutational burden (heteroplasmy level) with mtDNAcn and pathologies. Here, we defined a mtDNA heteroplasmic mutation as a point mutation or small indel with a relative frequency between 3 and $90 \%$. As in the previous section, we studied the four brain regions with $\geq 100$ samples and excluded the non-Caucasian samples from the MSBB dataset (a total of $n=1228$ brain samples). On average, between 2.6 and 2.8 heteroplasmic mutations were observed in the three cortical regions, whereas the $C B$ demonstrated less heteroplasmic mutations (mean of 1.0) (Fig. 4A). As expected, most of the heteroplasmic mutations were located in the mtDNA hypervariable control region, also known as the D-loop (Fig. 4B). None of the genes encoded in the mitochondrial genome showed an enrichment of heteroplasmic mutations.

Next, we used the DLPFC dataset to study whether the mtDNA heteroplasmy levels are related to brain pathologies, cognitive function, sex, age, and mtDNAcn. In contrast to the mtDNAcn, heteroplasmy levels were not associated with $\mathrm{AD}$ pathologies or cognitive function. We also found no significant association between heteroplasmy levels and mtDNAcn in the DLPFC. However, heteroplasmy levels increased significantly with advancing age: we report estimated $1.6 \%$ increase per year in the DLPFC ( $p=8.0 \times 10^{-6}$, quasi-Poisson regression) (Fig. 4C). The accumulation of mtDNA heteroplasmic mutations with aging in the cortex were replicated in the TCX and FP data with similar effect sizes (Fig. 4D-F). In line with the low abundance of heteroplasmic mutations in the $\mathrm{CB}$, the association with age was not detectable in this brain region. The association with age persisted when we adjusted for pathologic diagnosis, sex, and mtDNAcn using multivariable models for each of the three regions DLPFC, TCX, and FP (Fig. S4, Table S5). In addition, we found a weak association with $\mathrm{AD}$ diagnosis and with mtDNAcn in the TCX, which were absent in the DLPFC and in the FP. Overall, our analyses showed that age is the primary driver of mtDNA heteroplasmic point mutations and small indels in the cortical regions and that the $\mathrm{CB}$ demonstrates low mtDNA mutation rates.

\section{Altered mtDNAcn does not necessarily imply alteration in mitochondrial content}

A lower mtDNAcn in a cell can reflect a lower mtDNAcn per mitochondrion or a lower mitochondrial content in the cell. We therefore quantified the mitochondrial content in the DLPFC using mass spectrometry-based proteomics data for a subset of 156 subjects who have DLPFC mtDNAcn measurements. Abundances of 10 proteins specific for mitochondria selected from the Human Protein Atlas were quantified, and the median of the standardized protein values was used as a mitochondrial content score (Fig. S5A) [37].

Table 2 Effect of APOE \&4 genotype on mtDNAcn

\begin{tabular}{|c|c|c|c|c|c|c|c|}
\hline \multirow[t]{2}{*}{ Region/Study } & \multirow{2}{*}{$\begin{array}{l}\mathrm{N} \text { of } A P O E \\
\varepsilon 4 \\
\text { dosages } \\
(0,1,2)\end{array}$} & \multicolumn{3}{|c|}{ Unadjusted for pathology } & \multicolumn{3}{|c|}{ Adjusted for pathology* } \\
\hline & & $\beta$ & SE & $\mathrm{p}$ & $\beta$ & SE & $\mathrm{p}$ \\
\hline DLPFC/ROSMAP & $335,112,7$ & -0.364 & 0.099 & $2.8 \times 10^{-04}$ & -0.204 & 0.105 & 0.051 \\
\hline CB/ROSMAP & $182,56,4$ & -0.292 & 0.134 & 0.031 & -0.223 & 0.144 & 0.124 \\
\hline TCX/Mayo & $183,70,9$ & -0.280 & 0.114 & 0.015 & -0.126 & 0.121 & 0.301 \\
\hline FP/MSBB & $171,88,11$ & -0.158 & 0.106 & 0.137 & -0.048 & 0.104 & 0.642 \\
\hline Meta-analysis & $871,326,31$ & -0.275 & 0.056 & $8.0 \times 10^{-07}$ & -0.141 & 0.058 & 0.014 \\
\hline
\end{tabular}

*ROSMAP was adjusted by adding a quantitative score for amyloid and tau burden to the model. Mayo and MSBB were adjusted by adding the pathologic diagnosis to the model 


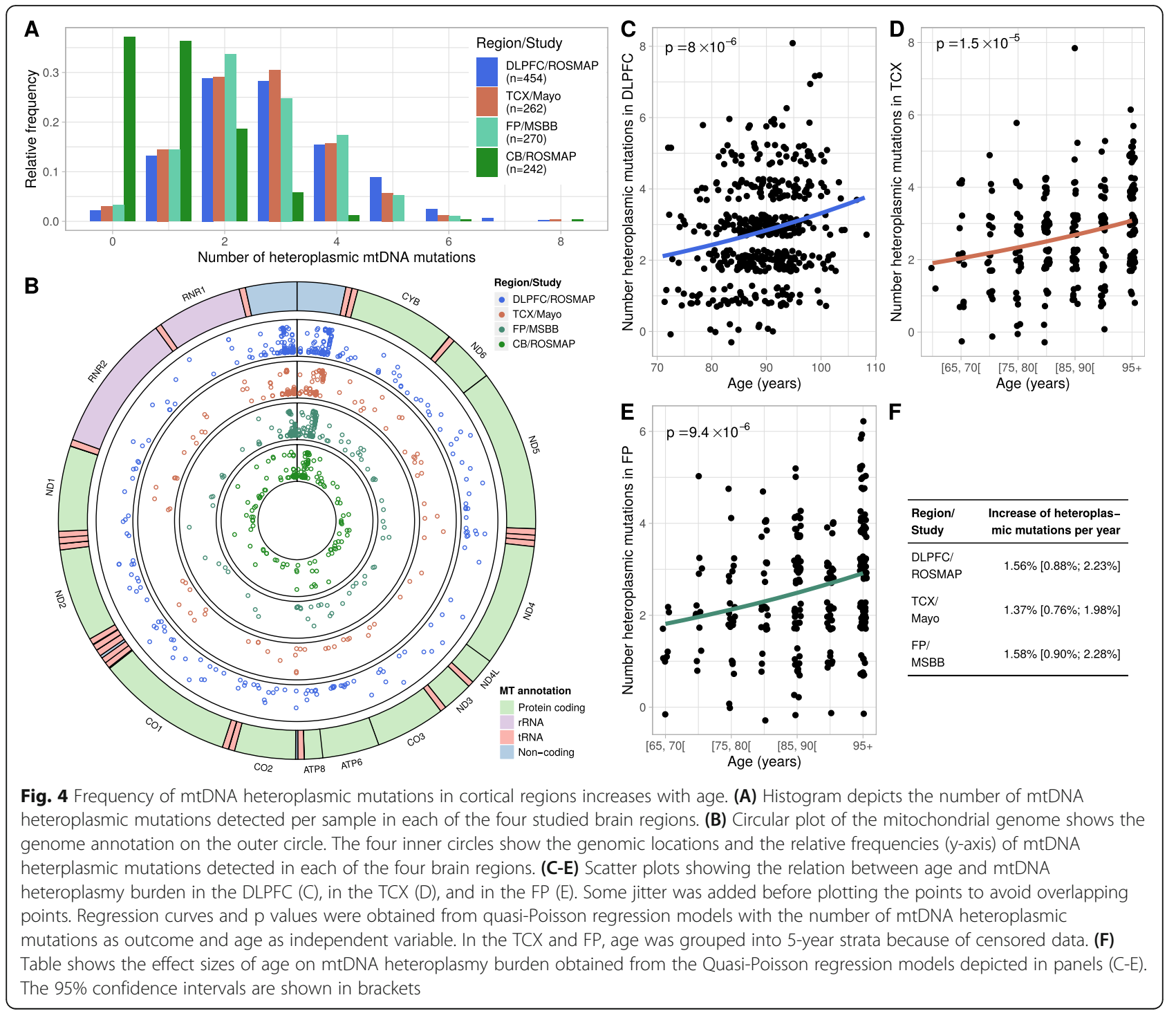

Interestingly, we found no significant correlation between mtDNAcn and mitochondrial content, but both measures were positively correlated with neuronal proportion and cognitive function (Fig. 5A, Fig. S5B). This suggests that these two measures (mtDNAcn and protein-based content) represent different aspects of mitochondrial health that both affect cognition but can change independently of each other. A similar lack of correlation between mtDNAcn and mitochondrial content has been previously reported for muscle tissue [58, 59]. Next, we derived a similar score using respiratory chain proteins encoded by the mitochondrial genome (data available for 11 of the 13 proteins, see methods, Supplementary Excel File 3) and observed a weak positive non-significant correlation of 0.11 with mtDNAcn (Fig. 5A), which is consistent with the notion that a moderate loss of mtDNA in not sufficient to reduce mitochondrial protein expression significantly. We also used our mass spectrometry data to quantify the abundances of respiratory chain complexes (see methods, Supplementary Excel File 3). Protein levels for complexes I to $\mathrm{V}$ were highly correlated with each other and with mitochondrial content, but demonstrated only a weak correlation with mtDNAcn (Fig. 5A). To further disentangle the relationship between mtDNAcn, mitochondrial content, mitochondrial heteroplasmy levels and AD-related phenotypes, we estimated the partial correlations between these variables. The graph in Fig. $5 \mathrm{~B}$ represents a sparse representation of the partial correlation matrix of the variables. An edge in this graph represents a direct association between two variables that remained when controlling for all other variables in the graph. The stability of the graph's edges was assessed using bootstrapping (Fig. S5C). The top left of the graph shows age as the primary driver for amyloid- $\beta$ and mtDNA heteroplasmy levels. The latter are not 


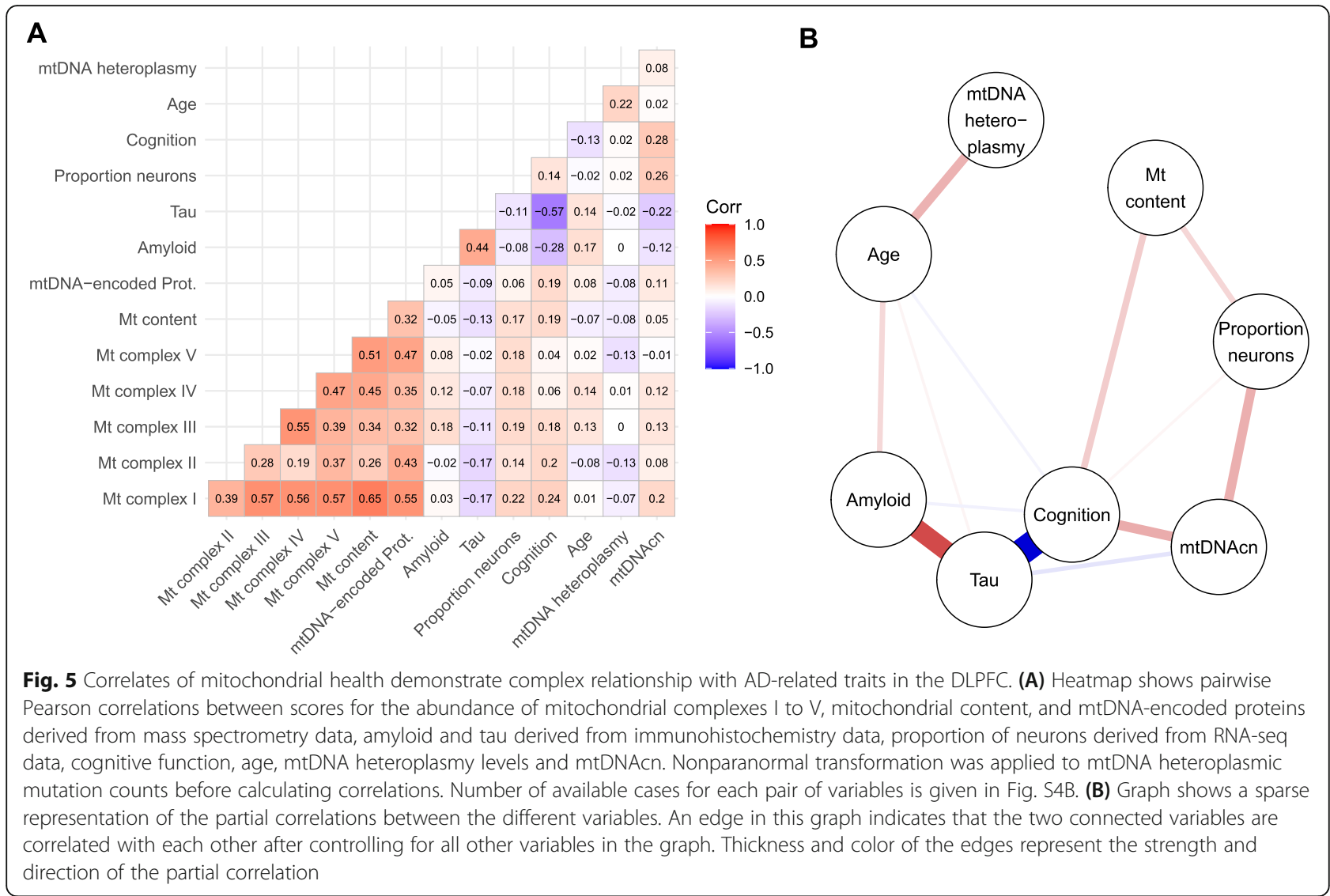

associated with any other variable in our graph suggesting that heteroplasmic mtDNA point mutations and small indels are not involved in AD pathogenesis. In the lower part of the graph, amyloid- $\beta$ is strongly associated with tau, and tau with cognitive function. Tau is also associated with mtDNAcn as suggested by our previous analyses. mtDNAcn and mitochondrial content are not directly connected, but both are associated with neuronal proportion and cognitive function. Together, these findings indicate a complex involvement of mitochondria in neurodegeneration and the need for future studies to measure both parameters to better understand mitochondrial recalibrations in AD.

\section{Discussion}

We characterized the mtDNAcn in 1361 aged human brain samples from five regions and estimated a reduction of mtDNAcn by 7 to $14 \%$ in pathologic AD compared to non-AD samples in the cortical regions profiled in this study. We then leveraged the detailed pathologic and cognitive characterization of the ROSMAP study to identify the primary drivers of mtDNAcn loss and to assess the relation to cognitive function in the presence of mixed pathologies, which are frequently observed in the aged human brain [20].

In the DLPFC, lower mtDNAcn was primarily associated with tau pathology. When accounting for ten common brain pathologies, tau was the only pathology that remained significantly associated with mtDNAcn. The mtDNAcn at the tissue level depends on the cell type composition of the studied tissue, as is well known in blood [54]. We therefore estimated the neuronal proportion in the DLPFC samples, confirmed that the neuronal proportion is associated with mtDNAcn, and demonstrated that the association between tau pathology and mtDNAcn was still significant - albeit attenuated - when adjusting for neuronal proportion. Although previous studies showed that informative estimates of neuronal proportions can be obtained from DLPFC RNA-seq data [60], we cannot exclude that changes in the complex composition of heterogeneous brain cells still contribute to the observed association. For example, a tau-mediated preferred depletion of a specific neuronal cell subtype could alter the composition within the neuronal cell population and cause changes in the mtDNAcn at the tissue level. Future single-cell studies will have to show to what extent the tau-related mtDNAcn loss observed 
in this study is a cell-intrinsic feature and which cell subtypes are affected.

Nevertheless, our analysis adjusted for neuronal proportions suggests that the relationship is not merely caused by tau-driven loss of mitochondria-rich neurons; other mechanisms link tau pathology to reduced mtDNAcn. Mechanisms that could potentially underlie our observation have been explored in model systems. For example, hyperphosphorylated tau has been shown to impair mitochondrial axonal transport $[61,62]$ and to affect mitochondrial fission/fusion dynamics [63, 64]. Conversely, reduced mtDNAcn has been shown to promote tau oligomerization in human neuronal cell lines [65], suggesting complex interactions between tau and mitochondria. Tau is also known to be a strong predictor for cognitive decline. Interestingly, when studying cognitive function, mtDNAcn was a significant predictor in our model that included tau, nine other brain pathologies, neuronal proportion, and demographic variables.

In contrast to tau pathology, amyloid- $\beta$ pathology was not significantly associated with mtDNAcn after accounting for other pathologies. This finding was supported by the results from the Mayo study where the mtDNAcn was not reduced in persons diagnosed with pathologic aging, which is defined by high amyloid- $\beta$ burden but no or minimal tau pathology [44]. Interestingly, numerous studies have demonstrated that the amyloid- $\beta$ precursor protein (APP) as well as amyloid- $\beta$ peptides localize at mitochondria and affect mitochondrial function and bioenergetics [66, 67]. Further, Cterminal fragments resulting from the processing of APP (APP-CTFs) have been implicated in AD and may trigger morphological and functional changes of mitochondria [68]. The absence of an association in our data could result from measuring only amyloid- $\beta$ peptides (1-40) and (1-42) (both were detected by our antibody), which does not fully capture APP processing. Further, amyloid- $\beta$ could still impair mitochondrial respiratory chain capacity or other non-energetic functions (e.g. calcium handling) without changes in mtDNAcn.

In the PCC, TDP-43 pathology was the most important factor and explained $23 \%$ of the mtDNAcn's variance. The effect of TDP-43 on mitochondria has been mainly studied in model systems for ALS, where suppressing the localization of TDP-43 inside mitochondria reduces TDP-43 toxicity [46]. When accumulating inside mitochondria, TDP-43 induces the release of mtDNA into the cytoplasm via the permeability transition pore [47]. Whether any of these mechanisms underlie the correlation observed in our post-mortem brain data remains to be elucidated. Interestingly, the effect of TDP43 pathology on mtDNAcn was moderate in the DLPFC, which could be caused by the distinct spatial pattern of TDP-43 progression in the aged brain or by a larger susceptibility of the neurons in the PCC to TDP- 43 pathology.

The mtDNAcn has been suggested as a biomarker for aging because several studies of peripheral blood have reported an inverse correlation between age and mtDNAcn $[69,70]$. However, more recent studies demonstrated that the inverse correlation is likely caused by unaccounted age-related changes of cell type proportions in the blood [71, 72]. In our study, we found no association between mtDNAcn and age or sex in the studied brain regions when we adjusted for pathologies. We also found no significant associations in the $C B$, which accumulates much less $\mathrm{AD}$ pathology than the DLPFC or the PCC. In summary, our results indicate that lower mtDNAcn is driven by certain pathologies rather than aging and restricted to brain regions directly affected by the respective pathologies. Similarly, a study of Parkinson's disease brains found lower mtDNAcn in the vulnerable substantia nigra but not in the frontal cortex, which is less affected in Parkinson's disease [73].

To assess the effect of genetic variants on the mtDNAcn in the brain, we performed a targeted analysis of 81 lead SNPs that were recently reported by a large GWAS of mtDNAcn in blood [52]. A missense variant in the protease LONP1 demonstrated a moderate but significant effect size of 0.23 standard deviations larger mtDNAcn per dosage of the alternative allele in our meta-analysis of four brain regions. The original bloodbased GWAS also detected a single variant at the $A P O E$ locus, which harbors the strongest genetic risk factor for $\mathrm{AD}$. We therefore investigated the effect of the $A P O E \& 4$ haplotype (defined by two coding variants) on the mtDNAcn. While a large fraction of the APOE $\varepsilon 4$ effect was mediated via $\mathrm{AD}$ pathologies, we also found evidence for a direct effect on the mtDNAcn. These findings support the hypothesis that the $A P O E \& 4$ allele exerts its risk not only via regulating amyloid- $\beta$ aggregation and clearance but also through other pathways, including mitochondrial bioenergetics [74, 75]. In summary, while this study is the first, to our knowledge, to report evidence for genetic regulation of the mtDNAcn in the brain, the sample size was a limiting factor and future non-targeted studies with much larger sample sizes will likely detect more loci.

Several tissues in addition to brain accumulate mtDNA mutations during aging [76-78]. Here, we analyzed point mutations and small indels and found higher heteroplasmy levels with age in three cortical regions and estimated an association consistent with an accumulation rate of about $1.5 \%$ per year in this age group. We did not find a significant association with age in the $\mathrm{CB}$ where the overall frequency of mtDNA heteroplasmic mutations was very low (mean of 1.0) supporting the theory of region-specific accumulation of mtDNA 
mutations in the brain [79]. Consistently, a previous study [9] failed to detect an association between age and heteroplasmy levels in their samples which were mainly (87\%) obtained from the CB. Thus, the CB seems to acquire less heteroplasmic mutations than the cortex or the heteroplasmic mutations in the CB have, on average, a lower relative frequency which may often not surpass the detection threshold of $3 \%$ used in this study. In contrast to the mtDNAcn, we found little evidence for the involvement of heteroplasmic mtDNA point mutations and small indels in AD or in the development of other brain pathologies. However, many of the previous studies reporting associations between mtDNA heteroplasmy and neurodegenerative diseases investigated structural variants, primarily large-scale mtDNA deletions, which were not considered in this study but are more likely to be pathogenic than small mutations [17, 18]. Overall, our work showed that small mtDNA heteroplasmic mutations accumulate over an individual's life-time in the cortex but are not related to neurodegenerative diseases. The seeming insignificance of low-level mtDNA heteroplasmy is consistent with a large reserve respiratory capacity, such that mtDNAcn is generally in excess $(>50 \%)$ of the minimum number of mtDNA copies required to sustain bioenergetic capacity [80].

A lower mtDNAcn can be caused by lower mitochondrial mass in the cells or by a lower mtDNAcn per mitochondrion. We generated a proteomic measure of mitochondrial content for a subset of our DLPFC samples and showed that the mtDNAcn and mitochondrial content vary relatively independent of each other in the DLPFC. The decoupling of the two variables may be explained by distinct mechanisms that regulate mtDNAcn and mitochondrial content and that have been successfully manipulated in model systems to regulate one of the readouts, without affecting the other one [5]. Similarly, the correlation between the abundance of mtDNAencoded proteins and mtDNAcn was weak, and protein measures of the respiratory chain complexes were highly correlated with each other and with mitochondrial content protein markers, but showed only weak correlations with mtDNAcn. Thus, the moderate reduction of mtDNAcn observed in this study may not have a functional effect on the respiratory chain capacity. Finally, when we integrated the detailed pathologic and cognitive measures with the mitochondrial measures available for our DLPFC samples to disentangle their relationship, we found that both, mtDNAcn and mitochondrial content, were associated with cognitive function and neuronal loss indicating their involvement in neurodegeneration and the need for future studies to interrogate several mitochondrial measures to fully capture mitochondrial health.

\section{Conclusions}

We profiled mtDNA quantity and quality across multiple regions in the aged human brain to study their relation with various age-related pathologies and cognitive function. Overall, lower mtDNAcn was found in regions affected by pathologies, and the loss of mtDNA was primarily related to tau and not to amyloid- $\beta$ pathology. The large effect of TDP-43 on mtDNAcn in the PCC points to a vulnerability of this region in LATE-NC. Our data further showed that the lower mtDNAcn is not just a consequence of neuronal loss, but directly related to worse cognitive function, and, thus, therapeutics enhancing mitochondrial function could potentially improve cognitive function. In the cortex, the frequency of heteroplasmic mtDNA point mutations and small indels increased with age, but there was little evidence pointing to an association with pathologies, suggesting that small heteroplasmic mtDNA mutations are probably not involved in the pathogenesis of age-related neurodegenerative diseases.

\section{Abbreviations}

AD: Alzheimer's disease; CB: Cerebellum; DLPFC: Dorsolateral prefrontal cortex; FP: Frontal pole; GWAS: Genome-wide association study; LATENC: limbic-predominant age-related TDP-43 encephalopathy neuropathological change; MSBB: Mount Sinai Brain Bank; mtDNA: Mitochondrial DNA; mtDNAcn: Mitochondrial DNA copy number; NUMT: Nuclear mitochondrial DNA; PCC: Posterior cingulate cortex; PD: Parkinson's disease; PSP: Progressive supranuclear palsy; ROSMAP: Religious Orders Study and Rush Memory and Aging Project; SNP: Single nucleotide polymorphism; TCX: Temporal cortex; TMT: Tandem mass tag; WGS: Whole-genome sequencing

\section{Supplementary Information}

The online version contains supplementary material available at https://doi. org/10.1186/s13024-021-00495-8.

Additional file 1. Table S1. Characteristics of the Mayo cohort. Table S2. Characteristics of the MSBB cohort. Table S3. Detailed results from the univariate regression analyses shown in Fig. 2A. Table S4.

Association between cell type proportions and mtDNAcn in the DLPFC. Table S5. Association between number of mtDNA heteroplasmic mutations and age adjusted for pathologic diagnosis. Table S6. Datasets and analysis results deposited at the AD Knowledge Portal. Fig. S1. Logtransformed mtDNAcn values are approximately normally distributed. Fig. S2. Changes of the mtDNAcn are primarily associated with tau in the DLPFC. Fig. S3. Significant fraction of the APOE $\varepsilon 4$ effect on mtDNAcn is mediated via AD pathologies. Fig. S4. Number of mtDNA heteroplasmic mutations in cortical regions is associated with age adjusted for sex, pathologic diagnosis, and mtDNAcn. Fig. S5. Mitochondrial content score and its relation to mtDNAcn, mtDNA heteroplasmy burden and AD-related phenotypes.

Additional file 2. Supplementary Excel File 1 contains DNA sequencing quality metrics, estimated raw and normalized mtDNAcn, estimated heteroplasmy levels, and phenotype information for all samples from the ROSMAP, Mayo Clinic, and MSBB studies.

Additional file 3. Supplementary Excel File 2 contains detailed results from the analysis of the 81 candidate SNPS and their effects on mtDNAcn.

Additional file 4. Supplementary Excel File 3 lists the proteins that were used to derive the protein scores for the respiratory chain complexes. 


\section{Acknowledgements}

Not applicable.

\section{Authors' contributions}

HUK, DAB and PLD conceptualized the study. DAB and PLD acquired funding and supervised the project. HUK analyzed the data and drafted the manuscript. AJL analyzed the RNA-seq data. HUK, CT, HSY, MP, DAB and PLD interpreted the results. All authors reviewed the manuscript and provided critical insights. All authors read and approved the final manuscript.

\section{Funding}

This work was supported by NIH grants U01AG046152 (PLD, DAB), U01AG061356 (PLD, DAB), P30AG010161 (DAB), R01AG015819 (DAB), and R01AG017917 (DAB)

\section{Availability of data and materials}

Raw and processed data (WGS, proteomics, RNA-seq), called variants, and analysis output (mtDNAcn, mtDNA heteroplasmy levels) from the three studies (see Table S6 for details) are available via the AD Knowledge Portal (https://adknowledgeportal.org). The AD Knowledge Portal is a platform for accessing data, analyses, and tools generated by the Accelerating Medicines Partnership (AMP-AD) Target Discovery Program and other National Institute on Aging (NIA)-supported programs to enable open-science practices and accelerate translational learning. The data, analyses and tools are shared early in the research cycle without a publication embargo on secondary use. Data is available for general research use according to the following requirements for data access and data attribution (https://adknowledgeportal.org/ DataAccess/Instructions)

For access to content described in this manuscript see: https://doi.org/10 7303/syn25618990.

Pathologic and phenotypic data from ROSMAP are available on our Resource Sharing Hub at https://www.radc.rush.edu.

$R$ and shell scripts used to generate figures and analysis results are deposited at GitHub: https://github.com/cu-ctcn/mtDNA.

\section{Declarations}

\section{Ethics approval and consent to participate}

The ROS and MAP studies were approved by the Institutional Review Board of Rush University Medical Center. All participants signed an informed consent, an Anatomic Gift Act for brain donation, and a repository consent to allow their data and biospecimens to be shared. The Mayo case-control study was approved by the Mayo Clinic Institutional Review Board. The MSBB study was approved by the Mount Sinai and JJ Peters VA Medical Center Institutional Review Boards.

\section{Consent for publication}

Not applicable.

\section{Competing interests}

The authors declare that they have no competing interests.

\section{Author details}

${ }^{1}$ Center for Translational \& Computational Neuroimmunology, Department of Neurology, Columbia University Irving Medical Center, New York, NY 10032, USA. ${ }^{2}$ Taub Institute for Research on Alzheimer's Disease and the Aging Brain, Columbia University Irving Medical Center, New York, NY 10032, USA. ${ }^{3}$ Division of Behavioral Medicine, Department of Psychiatry, Columbia University Irving Medical Center, New York, NY 10032, USA. ${ }^{4}$ Center for Alzheimer Research and Treatment, Department of Neurology, Brigham and Women's Hospital, Boston, MA 02115, USA. ${ }^{5}$ Merritt Center and Columbia Translational Neuroscience Initiative, Department of Neurology, Columbia University Irving Medical Center, New York, NY 10032, USA. 'Rush Alzheimer's Disease Center, Rush University Medical Center, Chicago, IL 60612, USA.

Received: 2 July 2021 Accepted: 11 October 2021

Published online: 06 November 2021

\section{References}

1. Nunnari J, Suomalainen A. Mitochondria: in sickness and in health. Cell. 2012;148(6):1145-59 https://doi.org/10.1016/j.cell.2012.02.035.
2. Spinelli JB, Haigis MC. The multifaceted contributions of mitochondria to cellular metabolism. Nat Cell Biol. 2018;20(7):745-54 https://doi.org/10.1038/ s41556-018-0124-1.

3. Sun N, Youle RJ, Finkel T. The mitochondrial basis of aging. Mol Cell. 2016; 61(5):654-66 https://doi.org/10.1016/j.molcel.2016.01.028.

4. Area-Gomez E, Guardia-Laguarta C, Schon EA, Przedborski S. Mitochondria, OxPhos, and neurodegeneration: cells are not just running out of gas. J Clin Invest. 2019;129(1):34-45 https://doi.org/10.1172/JCl120848.

5. Filograna R, Mennuni M, Alsina D, Larsson NG. Mitochondrial DNA copy number in human disease: the more the better? FEBS Lett. 2020. https://doi. org/10.1002/1873-3468.14021;595(8):976-1002.

6. D'Erchia AM, Atlante A, Gadaleta G, Pavesi G, Chiara M, De Virgilio C, et al. Tissue-specific mtDNA abundance from exome data and its correlation with mitochondrial transcription, mass and respiratory activity. Mitochondrion. 2015;20:13-21 https://doi.org/10.1016/j.mito.2014.10.005.

7. Stewart JB, Chinnery PF. Extreme heterogeneity of human mitochondrial DNA from organelles to populations. Nat Rev Genet. 2021;22(2):106-18 https://doi.org/10.1038/s41576-020-00284-x.

8. Coskun PE, Beal MF, Wallace DC. Alzheimer's brains harbor somatic mtDNA control-region mutations that suppress mitochondrial transcription and replication. Proc Natl Acad Sci U S A. 2004;101(29):10726-31 https://doi. org/10.1073/pnas.0403649101.

9. Wei W, Keogh MJ, Wilson I, Coxhead J, Ryan S, Rollinson S, et al. Mitochondrial DNA point mutations and relative copy number in 1363 disease and control human brains. Acta Neuropathol Commun. 2017;5(1):13 https://doi.org/10.1186/s40478-016-0404-6.

10. Rice AC, Keeney PM, Algarzae NK, Ladd AC, Thomas RR, Bennett JP Jr. Mitochondrial DNA copy numbers in pyramidal neurons are decreased and mitochondrial biogenesis transcriptome signaling is disrupted in Alzheimer's disease hippocampi. J Alzheimers Dis. 2014;40(2):319-30 https://doi.org/1 0.3233/JAD-131715.

11. Rodriguez-Santiago B, Casademont J, Nunes V. Is mitochondrial DNA depletion involved in Alzheimer's disease? Eur J Hum Genet. 2001;9(4):27985 https://doi.org/10.1038/sj.ejhg.5200629.

12. Yang SY, Castellani CA, Longchamps RJ, Pillalamarri VK, O'Rourke B, Guallar E, et al. Blood-derived mitochondrial DNA copy number is associated with gene expression across multiple tissues and is predictive for incident neurodegenerative disease. Genome Res. 2021;31(3):349-58 https://doi. org/10.1101/gr.269381.120.

13. Frahm T, Mohamed SA, Bruse $P$, Gemund $C$, Oehmichen M, Meissner $C$. Lack of age-related increase of mitochondrial DNA amount in brain, skeletal muscle and human heart. Mech Ageing Dev. 2005;126(11):1192-200 https:// doi.org/10.1016/j.mad.2005.06.008.

14. Miller FJ, Rosenfeldt FL, Zhang C, Linnane AW, Nagley P. Precise determination of mitochondrial DNA copy number in human skeletal and cardiac muscle by a PCR-based assay: lack of change of copy number with age. Nucleic Acids Res 2003;31(11):e61. https://doi.org/10.1093/nar/gng060, $61 e, 661$.

15. Wachsmuth M, Hubner A, Li M, Madea B, Stoneking M. Age-related and Heteroplasmy-related variation in human mtDNA copy number. PLoS Genet. 2016;12(3):e1005939 https://doi.org/10.1371/journal.pgen.1005939.

16. Larsson NG. Somatic mitochondrial DNA mutations in mammalian aging. Annu Rev Biochem. 2010;79(1):683-706. https://doi.org/10.1146/annurevbiochem-060408-093701.

17. Bender A, Krishnan KJ, Morris CM, Taylor GA, Reeve AK, Perry RH, et al. High levels of mitochondrial DNA deletions in substantia nigra neurons in aging and Parkinson disease. Nat Genet. 2006;38(5):515-7 https://doi.org/10.1038/ ng1769.

18. Corral-Debrinski M, Horton T, Lott MT, Shoffner JM, McKee AC, Beal MF, et al. Marked changes in mitochondrial DNA deletion levels in Alzheimer brains. Genomics. 1994;23(2):471-6 https://doi.org/10.1006/ geno.1994.1525.

19. Bennett DA, Buchman AS, Boyle PA, Barnes LL, Wilson RS, Schneider JA. Religious orders study and rush memory and aging project. J Alzheimers Dis. 2018;64(s1):S161-S89 https://doi.org/10.3233/JAD-179939.

20. Schneider JA, Arvanitakis Z, Bang W, Bennett DA. Mixed brain pathologies account for most dementia cases in community-dwelling older persons. Neurology. 2007;69(24):2197-204 https://doi.org/10.1212/01.wnl.0000271 090.28148.24.

21. Bennett DA, Schneider JA, Wilson RS, Bienias JL, Arnold SE. Neurofibrillary tangles mediate the association of amyloid load with clinical Alzheimer 
disease and level of cognitive function. Arch Neurol. 2004;61(3):378-84 https://doi.org/10.1001/archneur.61.3.378.

22. Bennett DA, Schneider JA, Tang Y, Arnold SE, Wilson RS. The effect of social networks on the relation between Alzheimer's disease pathology and level of cognitive function in old people: a longitudinal cohort study. Lancet Neurol. 2006;5(5):406-12 https://doi.org/10.1016/S1474-4422(06)70417-3.

23. Nag S, Yu L, Wilson RS, Chen EY, Bennett DA, Schneider JA. TDP-43 pathology and memory impairment in elders without pathologic diagnoses of AD or FTLD. Neurology. 2017;88(7):653-60 https://doi.org/10.1212/WNL. 0000000000003610.

24. Schneider JA, Arvanitakis Z, Yu L, Boyle PA, Leurgans SE, Bennett DA. Cognitive impairment, decline and fluctuations in older communitydwelling subjects with Lewy bodies. Brain. 2012;135(Pt 10):3005-14 https:// doi.org/10.1093/brain/aws234.

25. Love S, Chalmers K, Ince P, Esiri M, Attems J, Jellinger K, et al. Development, appraisal, validation and implementation of a consensus protocol for the assessment of cerebral amyloid angiopathy in post-mortem brain tissue. Am J Neurodegener Dis. 2014;3(1):19-32.

26. Boyle PA, Yu L, Nag S, Leurgans S, Wilson RS, Bennett DA, et al. Cerebral amyloid angiopathy and cognitive outcomes in community-based older persons. Neurology. 2015;85(22):1930-6 https://doi.org/10.1212/WNL. 0000000000002175.

27. Arvanitakis Z, Capuano AW, Leurgans SE, Buchman AS, Bennett DA, Schneider JA. The relationship of cerebral vessel pathology to brain microinfarcts. Brain Pathol. 2017;27(1):77-85 https://doi.org/10.1111/bpa.12365.

28. Buchman AS, Leurgans SE, Nag S, Bennett DA, Schneider JA. Cerebrovascular disease pathology and parkinsonian signs in old age. Stroke. 2011:42(11):3183-9 https://doi.org/10.1161/STROKEAHA.111.623462.

29. Arvanitakis Z, Leurgans SE, Barnes LL, Bennett DA, Schneider JA. Microinfarct pathology, dementia, and cognitive systems. Stroke. 2011;42(3):722-7 https://doi.org/10.1161/STROKEAHA.110.595082.

30. Nag S, Yu L, Capuano AW, Wilson RS, Leurgans SE, Bennett DA, et al. Hippocampal sclerosis and TDP-43 pathology in aging and Alzheimer disease. Ann Neurol. 2015;77(6):942-52 https://doi.org/10.1002/ana.24388.

31. Allen M, Carrasquillo MM, Funk C, Heavner BD, Zou F, Younkin CS, et al. Human whole genome genotype and transcriptome data for Alzheimer's and other neurodegenerative diseases. Sci Data. 2016;3(1):160089. https:// doi.org/10.1038/sdata.2016.89.

32. Wang M, Beckmann ND, Roussos P, Wang E, Zhou X, Wang Q, et al. The Mount Sinai cohort of large-scale genomic, transcriptomic and proteomic data in Alzheimer's disease. Sci Data. 2018;5(1):180185. https://doi.org/10.103 8/sdata.2018.185.

33. De Jager PL, Ma Y, McCabe C, Xu J, Vardarajan BN, Felsky D, et al. A multiomic atlas of the human frontal cortex for aging and Alzheimer's disease research. Sci Data. 2018;5(1):180142. https://doi.org/10.1038/sdata.2018.142.

34. Zhong Y, Wan YW, Pang K, Chow LM, Liu Z. Digital sorting of complex tissues for cell type-specific gene expression profiles. BMC Bioinformatics. 2013;14(1):89. https://doi.org/10.1186/1471-2105-14-89.

35. Wang X, Allen M, Li S, Quicksall ZS, Patel TA, Carnwath TP, et al. Deciphering cellular transcriptional alterations in Alzheimer's disease brains. Mol Neurodegener. 2020;15(1):38 https://doi.org/10.1186/s13024-020-00392-6.

36. Wingo AP, Fan W, Duong DM, Gerasimov ES, Dammer EB, Liu Y, et al. Shared proteomic effects of cerebral atherosclerosis and Alzheimer's disease on the human brain. Nat Neurosci. 2020;23(6):696-700 https://doi.org/10.103 8/s41593-020-0635-5.

37. Thul PJ, Akesson L, Wiking M, Mahdessian D, Geladaki A, Ait Blal H, et al. A subcellular map of the human proteome. Science. 2017;356(6340) https:// doi.org/10.1126/science.aal3321.

38. Imai $\mathrm{K}$, Keele L, Yamamoto T. Identification, inference and sensitivity analysis for causal mediation effects. Stat Sci. 2010;25(1):51-71. https://doi.org/1 0.1214/10-STS321.

39. Epskamp S, Borsboom D, Fried El. Estimating psychological networks and their accuracy: a tutorial paper. Behav Res Methods. 2018;50(1):195-212 https://doi.org/10.3758/s13428-017-0862-1.

40. Ding J, Sidore C, Butler TJ, Wing MK, Qian Y, Meirelles O, et al. Assessing mitochondrial DNA variation and copy number in lymphocytes of $\sim 2,000$ Sardinians using tailored sequencing analysis tools. PLoS Genet. 2015;11(7): e1005306 https://doi.org/10.1371/journal.pgen.1005306.

41. Longchamps RJ, Castellani CA, Yang SY, Newcomb CE, Sumpter JA, Lane J, et al. Evaluation of mitochondrial DNA copy number estimation techniques. PLoS One. 2020;15(1):e0228166 https://doi.org/10.1371/journal.pone.0228166.
42. Guo W, Jiang L, Bhasin S, Khan SM, Swerdlow RH. DNA extraction procedures meaningfully influence qPCR-based mtDNA copy number determination. Mitochondrion. 2009;9(4):261-5 https://doi.org/10.1016/j. mito.2009.03.003.

43. Nacheva E, Mokretar K, Soenmez A, Pittman AM, Grace C, Valli R, et al. DNA isolation protocol effects on nuclear DNA analysis by microarrays, droplet digital PCR, and whole genome sequencing, and on mitochondrial DNA copy number estimation. PLoS One. 2017;12(7):e0180467 https://doi.org/1 0.1371/journal.pone.0180467.

44. Murray ME, Dickson DW. Is pathological aging a successful resistance against amyloid-beta or preclinical Alzheimer's disease? Alzheimers Res Ther. 2014;6(3):24 https://doi.org/10.1186/alzrt254.

45. Wang W, Li L, Lin WL, Dickson DW, Petrucelli L, Zhang T, et al. The ALS disease-associated mutant TDP-43 impairs mitochondrial dynamics and function in motor neurons. Hum Mol Genet. 2013;22(23):4706-19 https:// doi.org/10.1093/hmg/ddt319.

46. Wang W, Wang L, Lu J, Siedlak SL, Fujioka H, Liang J, et al. The inhibition of TDP-43 mitochondrial localization blocks its neuronal toxicity. Nat Med. 2016:22(8):869-78 https://doi.org/10.1038/nm.4130.

47. Yu CH, Davidson S, Harapas CR, Hilton JB, Mlodzianoski MJ, Laohamonthonkul P, et al. TDP-43 triggers mitochondrial DNA release via mPTP to activate CGAS/STING in ALS. Cell. 2020;183(3):636-49 e18. https:/ doi.org/10.1016/..cell.2020.09.020.

48. Nelson PT, Dickson DW, Trojanowski JQ, Jack CR, Boyle PA, Arfanakis K, et al. Limbic-predominant age-related TDP-43 encephalopathy (LATE): consensus working group report. Brain. 2019;142(6):1503-27 https://doi.org/10.1093/bra in/awz099.

49. Botha H, Mantyh WG, Murray ME, Knopman DS, Przybelski SA, Wiste HJ, et al. FDG-PET in tau-negative amnestic dementia resembles that of autopsy-proven hippocampal sclerosis. Brain. 2018;141(4):1201-17 https:// doi.org/10.1093/brain/awy049.

50. Josephs KA, Murray ME, Whitwell JL, Tosakulwong N, Weigand SD, Petrucelli $L$, et al. Updated TDP-43 in Alzheimer's disease staging scheme. Acta Neuropathol. 2016;131(4):571-85 https://doi.org/10.1007/s00401-016-1537-1.

51. Nag S, Yu L, Boyle PA, Leurgans SE, Bennett DA, Schneider JA. TDP-43 pathology in anterior temporal pole cortex in aging and Alzheimer's disease. Acta Neuropathol Commun. 2018;6(1):33 https://doi.org/10.1186/s4 0478-018-0531-3.

52. Longchamps R, Yang S, Castellani C, Shi W, Lane J, Grove M, et al. Genomewide analysis of mitochondrial DNA copy number reveals multiple loci implicated in nucleotide metabolism, platelet activation, and megakaryocyte proliferation. bioRxiv. 2021;2021.01.25.428086. https://doi.org/10.1101/2021. 01.25.428086.

53. Hagg S, Jylhava J, Wang Y, Czene K, Grassmann F. Deciphering the genetic and epidemiological landscape of mitochondrial DNA abundance. Hum Genet. 2020. https://doi.org/10.1007/s00439-020-02249-w; 140(6):849-61.

54. Shim HB, Arshad O, Gadawska I, Cote HCF, Hsieh AYY. Platelet mtDNA content and leukocyte count influence whole blood mtDNA content. Mitochondrion. 2020;52:108-14 https://doi.org/10.1016/..mito.2020.03.001.

55. Bota DA, Davies KJ. Mitochondrial Lon protease in human disease and aging: including an etiologic classification of Lon-related diseases and disorders. Free Radic Biol Med. 2016;100:188-98 https://doi.org/10.1016/j. freeradbiomed.2016.06.031.

56. Area-Gomez E, Larrea D, Pera M, Agrawal RR, Guilfoyle DN, Pirhaji L, et al. APOE4 is associated with differential regional vulnerability to bioenergetic deficits in aged APOE mice. Sci Rep. 2020;10(1):4277 https://doi.org/10.1038/ s41598-020-61142-8.

57. Yin J, Reiman EM, Beach TG, Serrano GE, Sabbagh MN, Nielsen M, et al. Effect of ApoE isoforms on mitochondria in Alzheimer disease. Neurology. 2020;94(23):e2404-e11 https://doi.org/10.1212/WNL.0000000000009582.

58. Brinckmann A, Weiss C, Wilbert F, von Moers A, Zwirner A, StoltenburgDidinger $\mathrm{G}$, et al. Regionalized pathology correlates with augmentation of mtDNA copy numbers in a patient with myoclonic epilepsy with raggedred fibers (MERRF-syndrome). PLoS One. 2010;5(10):e13513 https://doi.org/1 0.1371/journal.pone.0013513.

59. Larsen S, Nielsen J, Hansen CN, Nielsen LB, Wibrand F, Stride N, et al. Biomarkers of mitochondrial content in skeletal muscle of healthy young human subjects. J Physiol. 2012;590(14):3349-60 https://doi.org/10.1113/ jphysiol.2012.230185.

60. Patrick E, Taga M, Ergun A, Ng B, Casazza W, Cimpean M, et al. Deconvolving the contributions of cell-type heterogeneity on cortical gene 
expression. PLoS Comput Biol. 2020;16(8):e1008120 https://doi.org/10.1371/ journal.pcbi.1008120.

61. Kopeikina KJ, Carlson GA, Pitstick R, Ludvigson AE, Peters A, Luebke Jl, et al. Tau accumulation causes mitochondrial distribution deficits in neurons in a mouse model of tauopathy and in human Alzheimer's disease brain. Am J Pathol. 2011;179(4):2071-82 https://doi.org/10.1016/j.ajpath.2011.07.004.

62. Shahpasand K, Uemura I, Saito T, Asano T, Hata K, Shibata K, et al. Regulation of mitochondrial transport and inter-microtubule spacing by tau phosphorylation at the sites hyperphosphorylated in Alzheimer's disease. Neurosci. 2012;32(7):2430-41 https://doi.org/10.1523/JNEUROSCI.5927-11.2 012.

63. Li XC, Hu Y, Wang ZH, Luo Y, Zhang Y, Liu XP, et al. Human wild-type fulllength tau accumulation disrupts mitochondrial dynamics and the functions via increasing mitofusins. Sci Rep. 2016;6(1):24756. https://doi.org/10.1038/ srep24756.

64. Kandimalla R, Manczak M, Fry D, Suneetha Y, Sesaki H, Reddy PH. Reduced dynamin-related protein 1 protects against phosphorylated tau-induced mitochondrial dysfunction and synaptic damage in Alzheimer's disease. Hum Mol Genet. 2016;25(22):4881-97 https://doi.org/10.1093/hmg/ddw312.

65. Weidling IW, Wilkins HM, Koppel SJ, Hutfles L, Wang X, Kalani A, et al. Mitochondrial DNA manipulations affect tau oligomerization. J Alzheimers Dis. 2020;77(1):149-63 https://doi.org/10.3233/JAD-200286.

66. Devi L, Prabhu BM, Galati DF, Avadhani NG, Anandatheerthavarada HK. Accumulation of amyloid precursor protein in the mitochondrial import channels of human Alzheimer's disease brain is associated with mitochondrial dysfunction. J Neurosci. 2006;26(35):9057-68 https://doi.org/1 0.1523/JNEUROSCI.1469-06.2006.

67. Du H, Guo L, Fang F, Chen D, Sosunov AA, McKhann GM, et al. Cyclophilin $D$ deficiency attenuates mitochondrial and neuronal perturbation and ameliorates learning and memory in Alzheimer's disease. Nat Med. 2008; 14(10):1097-105 https://doi.org/10.1038/nm.1868.

68. Vaillant-Beuchot L, Mary A, Pardossi-Piquard R, Bourgeois A, Lauritzen I, Eysert $F$, et al. Accumulation of amyloid precursor protein C-terminal fragments triggers mitochondrial structure, function, and mitophagy defects in Alzheimer's disease models and human brains. Acta Neuropathol. 2021; 141(1):39-65 https://doi.org/10.1007/s00401-020-02234-7.

69. Fries GR, Bauer IE, Scaini G, Wu MJ, Kazimi IF, Valvassori SS, et al. Accelerated epigenetic aging and mitochondrial DNA copy number in bipolar disorder. Transl Psychiatry. 2017;7(12):1283 https://doi.org/10.1038/s41398-017-0048-8.

70. Mengel-From J, Thinggaard M, Dalgard C, Kyvik KO, Christensen K. Christiansen L. Mitochondrial DNA Copy number in peripheral blood cells declines with age and is associated with general health among elderly. Hum Genet. 2014;133(9):1149-59 https://doi.org/10.1007/s00439-014-1458-9.

71. Rausser S, Trumpff C, McGill MA, Junker A, Wang W, Ho S, et al. Mitochondrial phenotypes in purified human immune cell subtypes and cell mixtures. bioRxiv. 2021:2020.10.16.342923. https://doi.org/10.1101/2020.10.1 6.342923.

72. Moore AZ, Ding J, Tuke MA, Wood AR, Bandinelli S, Frayling TM, et al. Influence of cell distribution and diabetes status on the association between mitochondrial DNA copy number and aging phenotypes in the InCHIANTI study. Aging Cell. 2018;17(1) https://doi.org/10.1111/acel.12683.

73. Pyle A, Anugrha H, Kurzawa-Akanbi M, Yarnall A, Burn D, Hudson G. Reduced mitochondrial DNA copy number is a biomarker of Parkinson's disease. Neurobiol Aging. 2016;38:216 e7- e10. https://doi.org/10.1016/j. neurobiolaging.2015.10.033.

74. Liu CC, Liu CC, Kanekiyo T, Xu H, Bu G. Apolipoprotein E and Alzheimer disease: risk, mechanisms and therapy. Nat Rev Neurol. 2013;9(2):106-18 https://doi.org/10.1038/nrneurol.2012.263.

75. Huang Y. Abeta-independent roles of apolipoprotein E4 in the pathogenesis of Alzheimer's disease. Trends Mol Med. 2010;16(6):287-94 https://doi.org/1 0.1016/j.molmed.2010.04.004

76. Li M, Schroder R, Ni S, Madea B, Stoneking M. Extensive tissue-related and allele-related mtDNA heteroplasmy suggests positive selection for somatic mutations. Proc Natl Acad Sci U S A. 2015;112(8):2491-6 https://doi.org/10.1 073/pnas.1419651112.

77. Zhang R, Wang Y, Ye K, Picard M, Gu Z. Independent impacts of aging on mitochondrial DNA quantity and quality in humans. BMC Genomics. 2017; 18(1):890 https://doi.org/10.1186/s12864-017-4287-0.

78. Lin MT, Simon DK, Ahn CH, Kim LM, Beal MF. High aggregate burden of somatic mtDNA point mutations in aging and Alzheimer's disease brain. Hum Mol Genet. 2002;11(2):133-45 https://doi.org/10.1093/hmg/11.2.133.
79. Keogh MJ, Chinnery PF. Mitochondrial DNA mutations in neurodegeneration. Biochim Biophys Acta. 2015;1847(11):1401-11 https:/ doi.org/10.1016/j.bbabio.2015.05.015.

80. Rossignol R, Faustin B, Rocher C, Malgat M, Mazat JP, Letellier T. Mitochondrial threshold effects. Biochem J. 2003;370(Pt 3):751-62 https:// doi.org/10.1042/BJ20021594.

\section{Publisher's Note}

Springer Nature remains neutral with regard to jurisdictional claims in published maps and institutional affiliations.

\section{Ready to submit your research? Choose BMC and benefit from:}

- fast, convenient online submission

- thorough peer review by experienced researchers in your field

- rapid publication on acceptance

- support for research data, including large and complex data types

- gold Open Access which fosters wider collaboration and increased citations

- maximum visibility for your research: over $100 \mathrm{M}$ website views per year

At BMC, research is always in progress.

Learn more biomedcentral.com/submissions 